\title{
Squinting at Power Series
}

\author{
M. Douglas McIlroy \\ AT\&T Bell Laboratories \\ Murray Hill, New Jersey 07974
}

\begin{abstract}
Data streams are an ideal vehicle for handling power series. Stream implementations can be read off directly from simple recursive equations that define operations such as multiplication, substitution, exponentiation, and reversion of series. The bookkeeping that bedevils these algorithms when they are expressed in traditional languages is completely hidden when they are expressed in stream terms. Communicating processes are the key to the simplicity of the algorithms. Working versions are presented in newsqueak, the language of Pike's "squint'" system; their effectiveness depends critically on the stream protocol.
\end{abstract}

\section{Series and streams}

Power series are natural objects for stream processing. Pertinent computations are neatly describable by recursive equations. CSP (communicating sequential process) languages 1,2 are good vehicles for implementation. This paper develops the algorithmic ideas and reduces them to practice in a working CSP language, ${ }^{3}$ not coincidentally illustrating the utility of concurrent programming notions in untangling the logic of some intricate computations on sequences.

This elegant approach to power series computation, first demonstrated but never published by Kahn and MacQueen as an application of their data stream system, is still little known. ${ }^{4}$ Power series are represented as streams of coefficients, the exponents being given implicitly by ordinal position. (This is an infinite analogue of the familiar representation of a polynomial as an array of coefficients.) Thus the power series for the exponential function

$$
e^{x}=\sum_{n=0}^{\infty} x^{n} / n !=1+x+\frac{1}{2} x^{2}+\frac{1}{6} x^{3}+\frac{1}{24} x^{4}+\cdots
$$


is represented as a stream of rationals:

$$
1,1, \frac{1}{2}, \frac{1}{6}, \frac{1}{24}, \cdots
$$

Throughout the paper power series will be denoted by symbols such as $F$, with the functional form $F(x)$ being used when the variable must be made explicit. Subscripts denote individual coefficients:

$$
F(x)=\sum_{i=0}^{\infty} F_{i} x^{i}
$$

The corresponding stream will be referred to by the same symbol in program font: F. In programs the names of power series variables contain capital letters; scalars are all lower case. When new power series are calculated from old, the input series will always be called $F$ and $G$.

In this section all programs are written in pseudocode; later they will be translated into the language "newsqueak" to be run in the "squint" system.* The methods are strictly formal; analytic interpretation of results depends on the convergence of the power series in question. Thus assertions such as $\sum_{i} x^{i}=1 /(1-x)$ are understood to hold only where the series converge.

Addition. The sum, $S=F+G$, is easy. Since $S_{i}=F_{i}+G_{i}$, all we need is a looping process, which gets pairs of coefficients from the two inputs and puts their sum on the output S. The following pseudocode suffices.

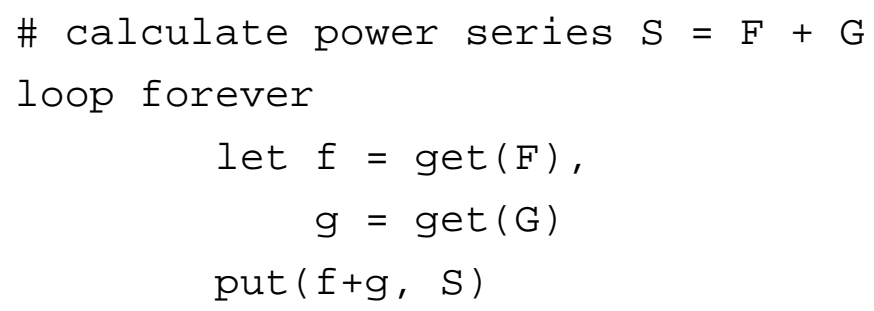

Multiplication by a term. Simple termwise operations on single power series, such as multiplication by a constant, integration, or differentiation are equally easy to program and are left to the reader's imagination. Multiplication of a power series by $x$ to yield $P=x F$ involves a one-element delay:

* The name of the language reveals descent from a mousier ancestor intended for programming a terminal with multiple asynchronous windows. The name "squint" suggests "squeak interpreter." 
\# calculate power series $P=x * F$

put $(0, \mathrm{P})$

loop forever

put (get $(F), P$ )

Multiplication. Calculation of the product, $P=F G$, is more challenging. By equating coefficients in

$$
\sum_{n=0}^{\infty} P_{n} x^{n}=\left(\sum_{i=0}^{\infty} F_{i} x^{i}\right)\left(\sum_{j=0}^{\infty} G_{j} x^{j}\right),
$$

we obtain the familiar convolution for terms of the product,

$$
P_{n}=\sum_{i=0}^{n} F_{i} G_{n-i}
$$

a most unpromising formula to program directly. To calculate the $n$th coefficient we must have stored up the first $n$ terms of both inputs, thus sacrificing most benefits of the stream representation. It will be best to look in another direction, guided by the adage: When iteration gets sticky, try recursion.

We treat streams recursively, as we do lists, by handling the leading term and recurring on the tail. This means rewriting a power series as a first term plus $x$ times another power series:

$$
F=F_{0}+x \bar{F}
$$

The tail $\bar{F}$ is a whole power series beginning with a constant term; it is ripe for recursion.

Writing the multiplication $P=F G$ recursively, we obtain

$$
P_{0}+x \bar{P}=F_{0} G_{0}+x\left(F_{0} \bar{G}+G_{0} \bar{F}\right)+x^{2} \bar{F} \bar{G}
$$

Equate coefficients to find the first term of the product,

$$
P_{0}=F_{0} G_{0}
$$

leaving for the tail,

$$
\bar{P}=F_{0} \bar{G}+G_{0} \bar{F}+x \bar{F} \bar{G}
$$

a sum of a constant $F_{0}$ times a power series, another constant $G_{0}$ times another power series, and $x$ times a third power series, which is itself a product $\bar{F} \bar{G}$. We already know 
how to multiply power series by a constant and how to add power series. We also know how to multiply power series by $x$; recursion does the rest.

In the following pseudocode, $\mathrm{f}{ }^{\star} \mathrm{G}$ denotes an auxiliary stream for the product $f G$, and so on. Each auxiliary stream will be computed by another process. Thus $f G$ will be computed by a process that multiplies a power series by a constant. When two auxiliary streams share an input stream, both see every element of the input.

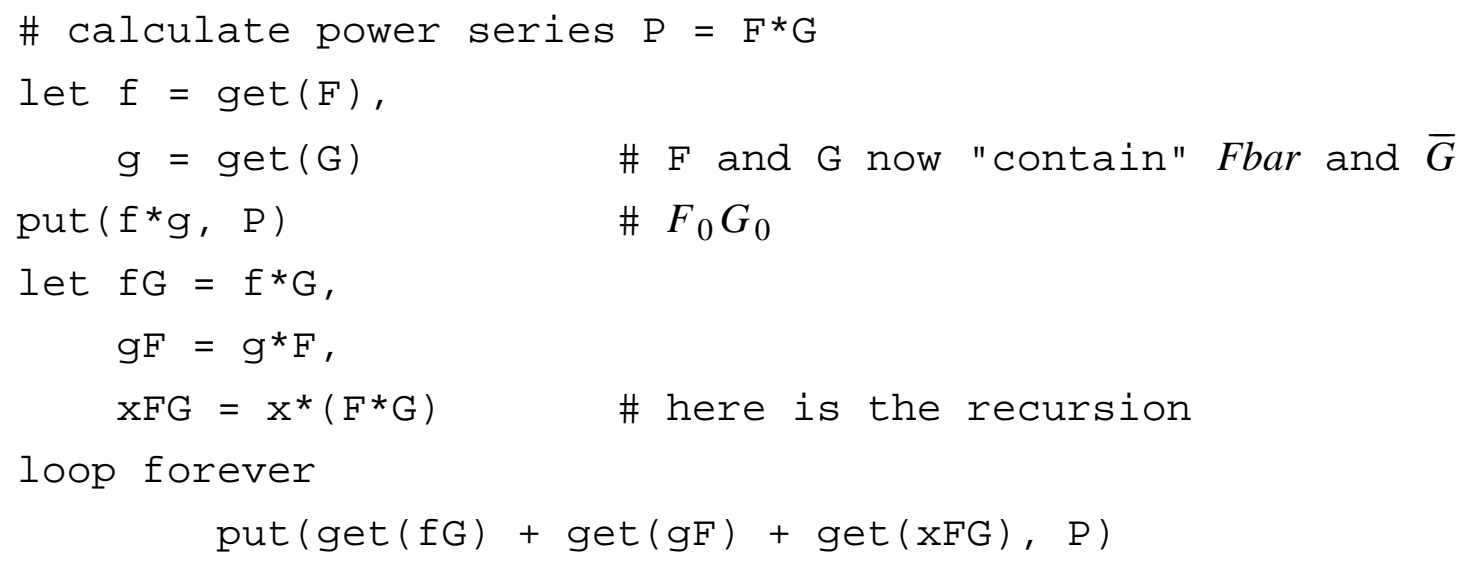

The convolution (1) has been hidden completely; all the necessary bookkeeping and storage management is embodied in the topology and contents of the auxiliary streams. Streams fan out to feed multiple consumers. For instance $G$ enters into both $f \star_{G}$ and $\mathrm{F}^{\star} \mathrm{G}$. The topology ramifies as the recursion progresses; more auxiliary streams appear at every stage, making the recursive picture of Figure 1. To write the program, though, we need not think about the topology, for the program is a direct image of the mathematics (2), and the mathematics is simple.

Substitution. Substitution, or composition, of power series, defined by $S=F(G)$, may be done similarly, using multiplication as a subprocess. The recursive formulation is

$$
S_{0}+x \bar{S}=F_{0}+\left(G_{0}+x \bar{G}\right) \bar{F}\left(G_{0}+x \bar{G}\right) .
$$

Equate coefficients to find the constant term,

$$
S_{0}=F_{0}+G_{0} \cdot(\text { first term of } \bar{F}(G)) \text {. }
$$

Induction shows that in general $S_{0}$ is an infinite sum.

$$
S_{0}=\sum_{i=0}^{\infty} F_{i} G_{0}^{i}
$$

To keep the problem finite, we further stipulate that $G_{0}=0$. Now 


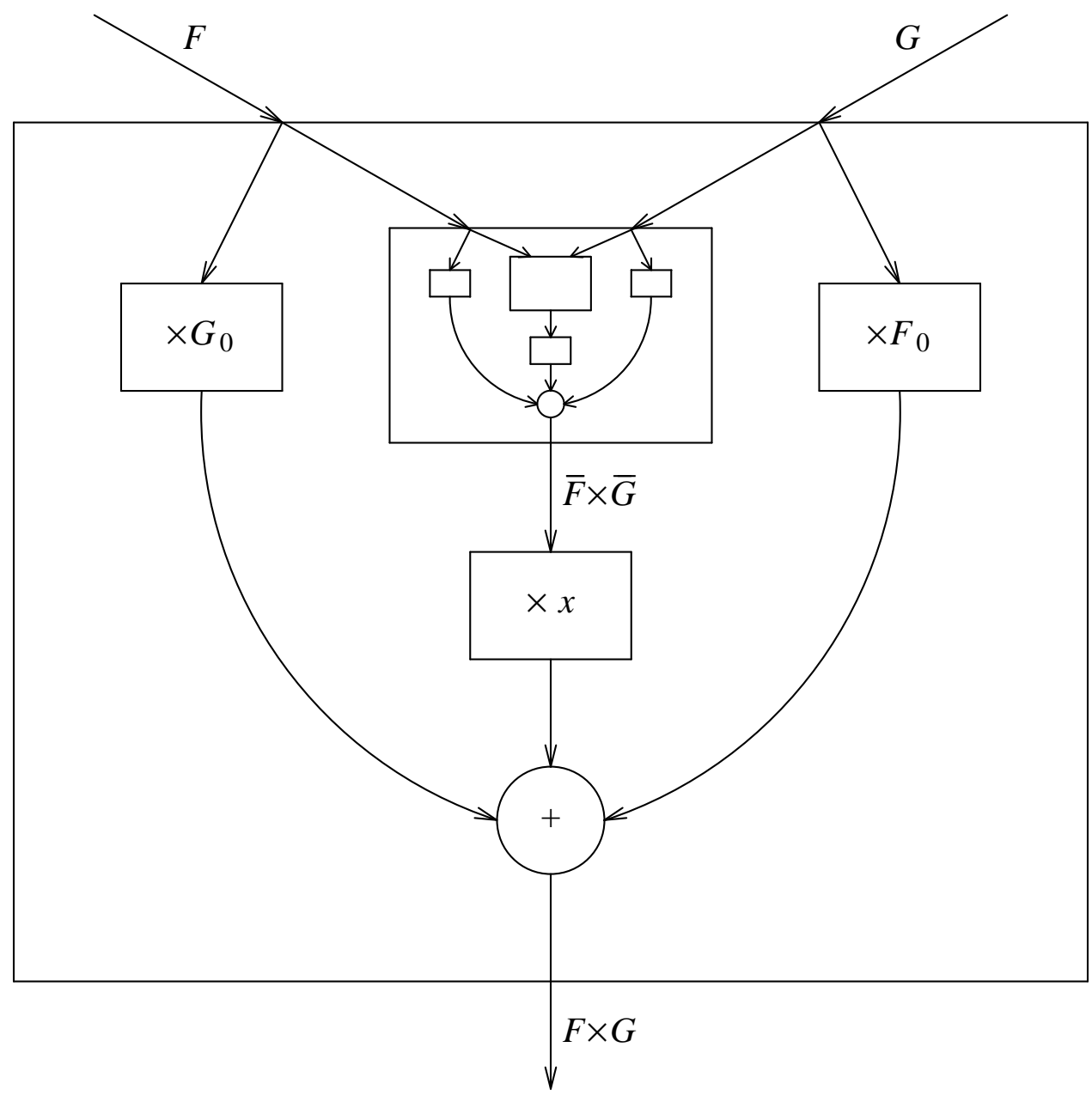

Figure 1. Data flow for multiplication. The outer box represents the process for the tail of $F G$, equation (2). The similar inner box does not receive the first terms of $F$ or $G$, and its output does not enter into the first two terms of the product $F G$. The newsqueak implementation uses a separate process for each box, and one or more processes for each stream splitting.

Viewed as a three-dimensional scene receding into the distance, the picture shows a simple repeating structure connected on top by $F$ and $G$ buses. The buses grow, extending one level further with the receipt of each input term. A daisy chain of output connections leads from back to front.

$$
S=S_{0}+x \bar{S}=F_{0}+x \bar{G} \bar{F}(G),
$$

from which pseudocode can be read off. 


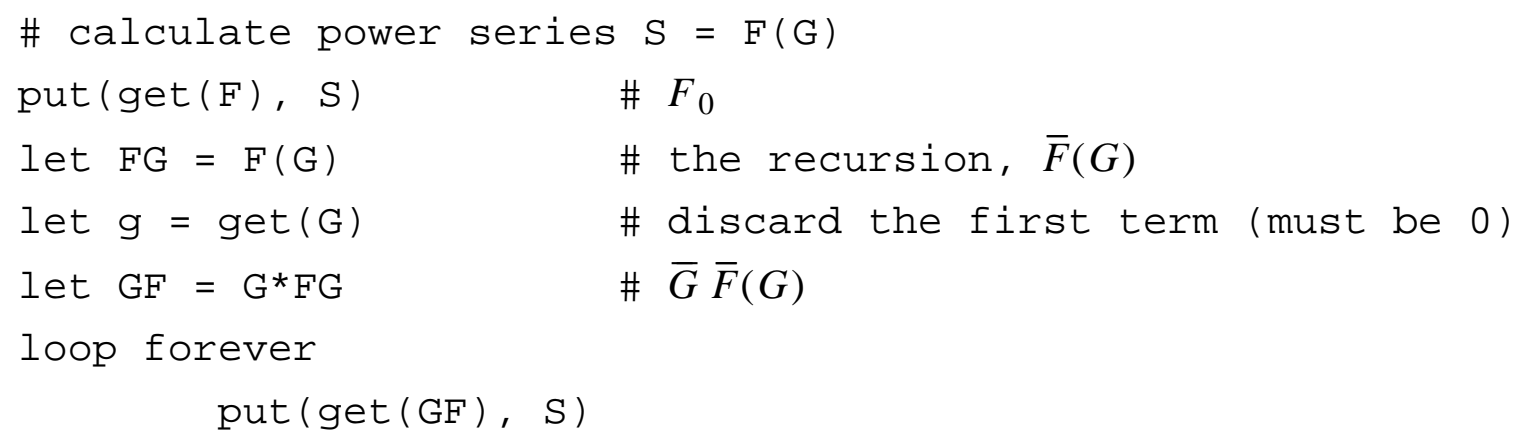

A subtlety: the $G$ in let $F G=F(G)$ stands for the whole power series, while the $G$ in let $\mathrm{GF}=\mathrm{G}^{\star} \mathrm{FG}$ stands only for the tail $\bar{G}$. In the same way, the meaning of $\mathrm{F}$ changes between get $(F)$ and let $F G=F(G)$.

Exponentiation. We wish to compute $X=e^{F}$. From the recursive representation,

$$
X=e^{F_{0}} e^{x \bar{F}}
$$

we see that

$$
X=e^{F_{0}} \cdot(1+\text { terms in } x) .
$$

To make the constant term of $X$ rational, we further require that $F_{0}=0$. Hence $X_{0}=1$.

Unfortunately equation (4) does not lead to an algorithm. Formal substitution yields

$$
X(x)=1 \cdot X(x \bar{X})
$$

Since the first term of $X(x)$ depends on the first term of $X(x \bar{X})$, we are stuck in an infinite regress. By induction we may conclude that the first term is an infinite product of ones, but what are the higher terms? We can "cheat," and substitute $F$ in the known power series for the exponential. It is more satisfying, though, to build from nothing and let the program "discover'" the exponential for itself.

A neat trick suffices. The identity,

$$
X^{\prime}=\frac{d}{d x} e^{F}=e^{F} F^{\prime}=X F^{\prime},
$$

may be read as a differential equation for $X$, with the formal solution,

$$
X(x)=\int_{0}^{x} X(t) F^{\prime}(t) d t+c
$$


where the constant of integration $c$ is the constant term $X_{0}=1$. Now we can sketch the program.

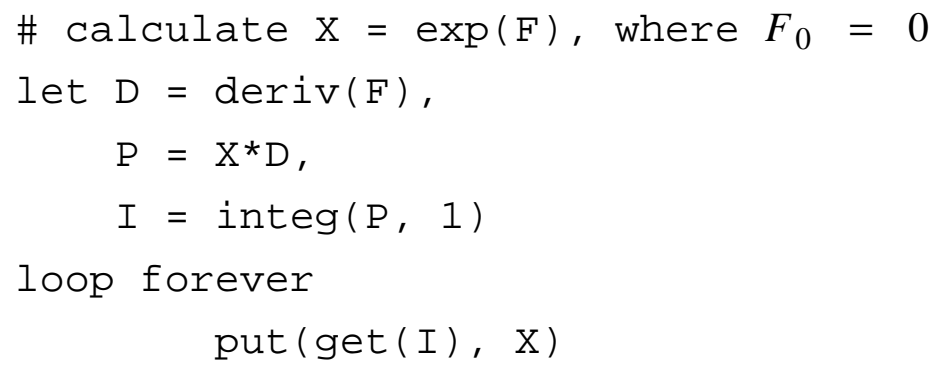

Here the stream operator deriv differentiates and the operator integ integrates with a given constant of integration. The calculation involves a data stream loop: $\mathrm{X}$ enters as an input into the calculation of $\mathrm{X}$. Unlike the recursion in equation (5), this self-reference is benign. Deadlock is avoided because the integral operator produces the first term of its output (the constant of integration) before it needs any input. This result gets fed onto stream $\mathrm{X}$ to help calculate the next term, and so on.

The method of calculating power series by integration feeding on itself has a distinguished lineage. It may be recognized as an instance of the classical Picard method of successive approximations for solving $y^{\prime}=f(x, y) .{ }^{5}$ It was first used in stream context by Kahn and MacQueen. Abelson and Sussman give the degenerate case of $\exp (x){ }^{6}$

Evidently the network of streams for calculating a product or an exponential entails careful scheduling. Certain lazy-evaluation systems, such as Miranda, handle such scheduling invisibly. ${ }^{7}$ For example, in terms of lazily-evaluated infinite lists, we may write the following functional pseudocode for power-series addition and multiplication.

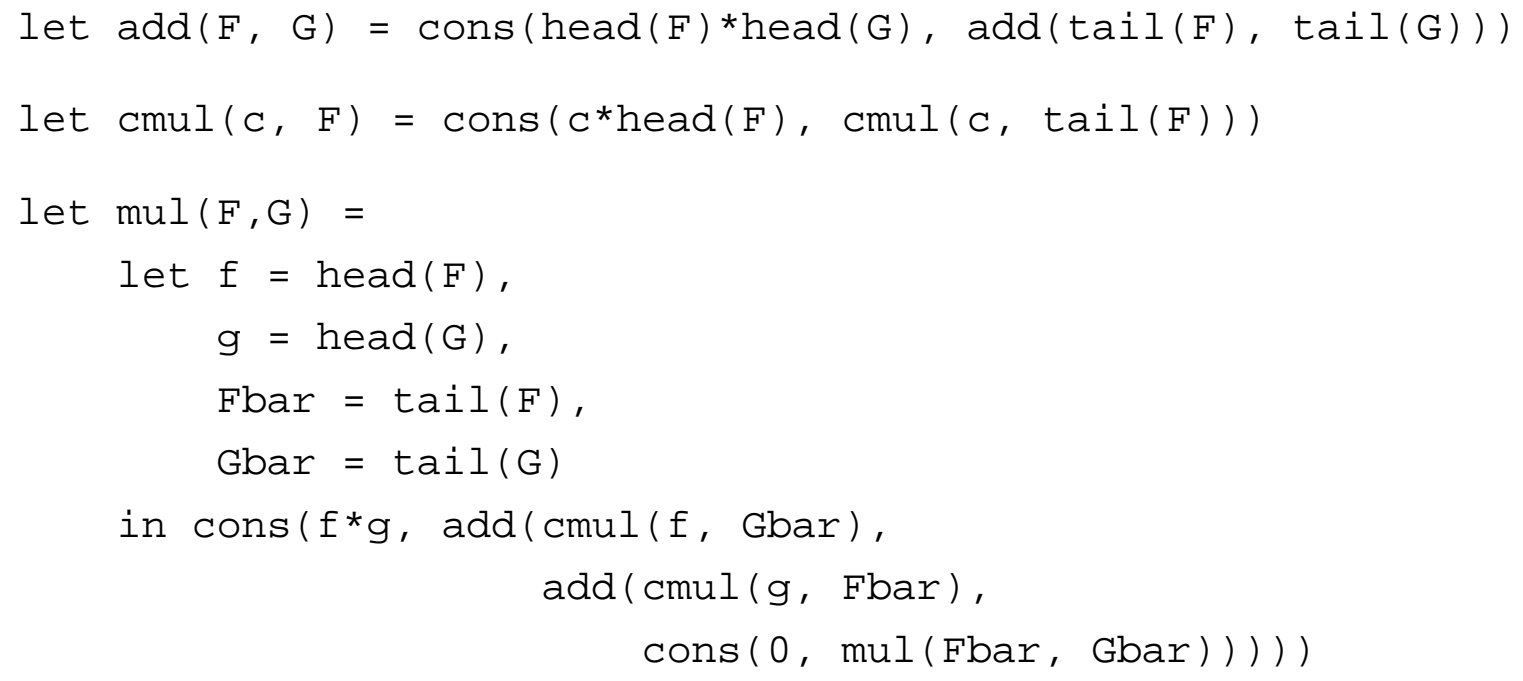


These functions follow from the same recursive equations as did the previous pseudocode, with head and cons playing the role of get and put.

Lazily-evaluated languages are, alas, not widely disseminated. Moreover, they do not inherently offer control over the exploitation of parallelism. An alternative exists in CSP languages. These languages require some infrastructure to be built to handle the scheduling. Once that is done, it becomes possible to write more efficient (though more verbose) code in CSP style. We shall return to this comparison at the end of the paper. In the meantime we proceed to an implementation in a channel-based CSP language.

\section{Translation to newsqueak}

Pike's newsqueak language supports asynchronous processes communicating over typed channels. Channels and functions are first-class citizens. Values of both kinds may be assigned to variables, passed as arguments, and returned by functions. Processes and channels may be created at will. There is just one kind of interprocess communication-rendezvous of two processes on a channel. Consequently the queuing and fanout that are necessary for power series computations must be programmed explicitly. A fair choice is made among competing rendezvous. When not waiting for rendezvous, all processes progress, at dithered rates, a few atomic operations per interpreter cycle.

Most of newsqueak will be familiar to experienced programmers. The communication semantics descends mainly from CSP, ${ }^{1}$ the syntax from $\mathrm{C}$, and the type system from Pascal and ML. ${ }^{8}$ Unusual features will be described as they are needed. For details see the accompanying paper and the manual. ${ }^{3,9}$

At base we need rationals, declared as numerator-denominator pairs, and power series, declared as channels that carry rationals,

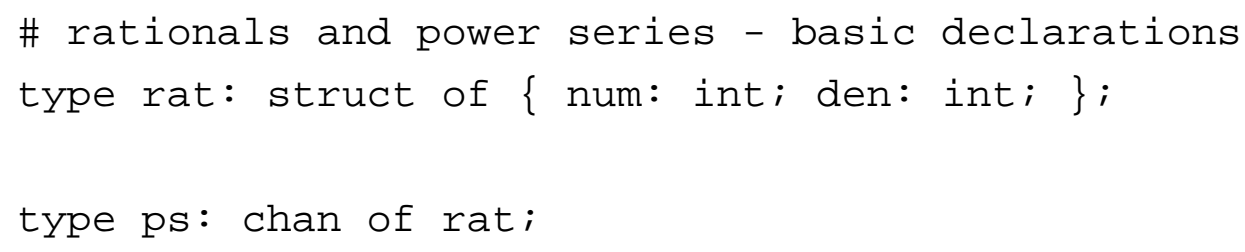

along with some obvious support routines, declared as follows. The literal definitions of these functions, all one-liners, have been left out, simply to avoid discussing the finicky, but straightforward, matter of data constructors. 


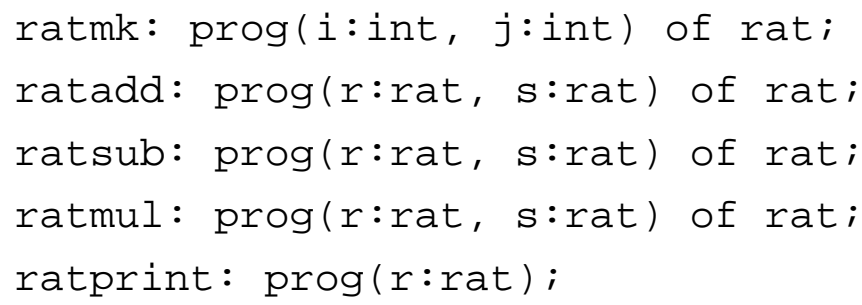

psmk: prog() of ps;

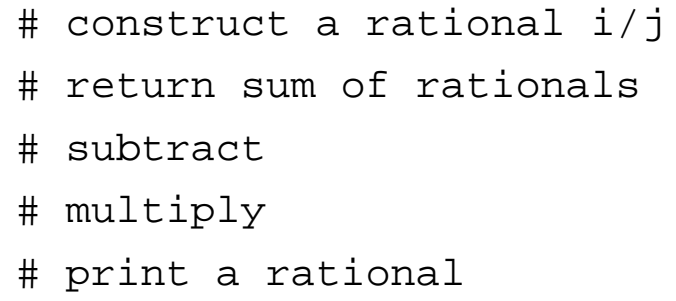

\# construct a power series

A program to print (endlessly) a power series is easy. To write it, though, we need some peculiar newsqueak syntax. Beware, in newsqueak $:=$ is not a simple assignment. It declares and initializes a new variable of a type inferred from the initializing expression. Assignment is represented by $=$ as in $\mathrm{C}$. The prefix operator $<-$ corresponds to get. A prog declarator followed by a body in braces \{\} denotes a value of function or procedure type. The printing program, which never terminates, is a procedure. It is given as the initial value of psprint, which consequently becomes a procedure.

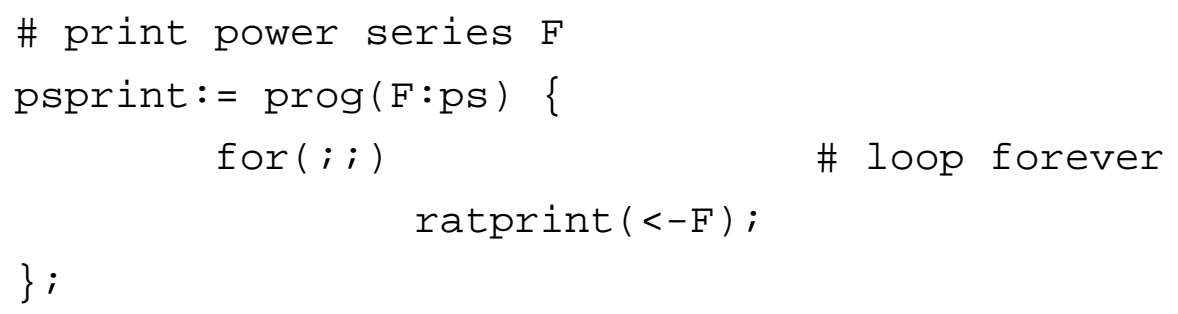

Adding power series is not much harder. We need one more operator, $<-=$, which plays the role of put.

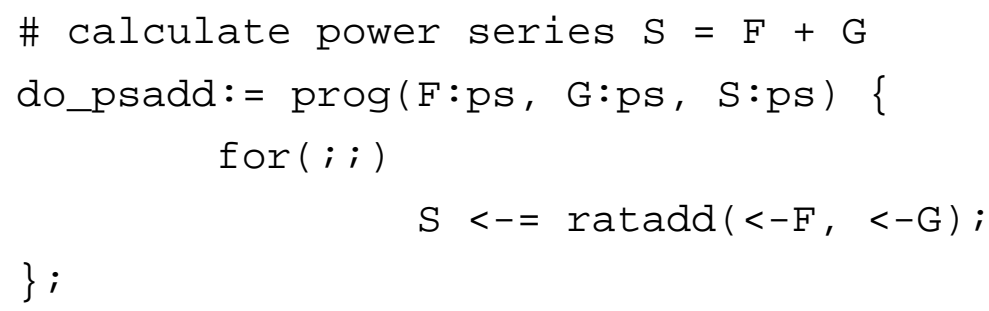

This procedure must be run as a separate process, which we do by invoking it in a begin statement. $\dagger$

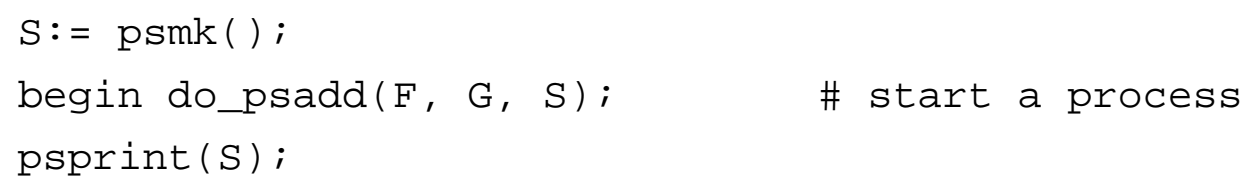

$\dagger$ In newsqueak begin is a verb, not punctuation as in Pascal. It serves the same purpose as postfix \& in UNIX® shells. 
The usage can be prettied up by encapsulating (and dropping the name of) do_psadd in a function that returns a power series.

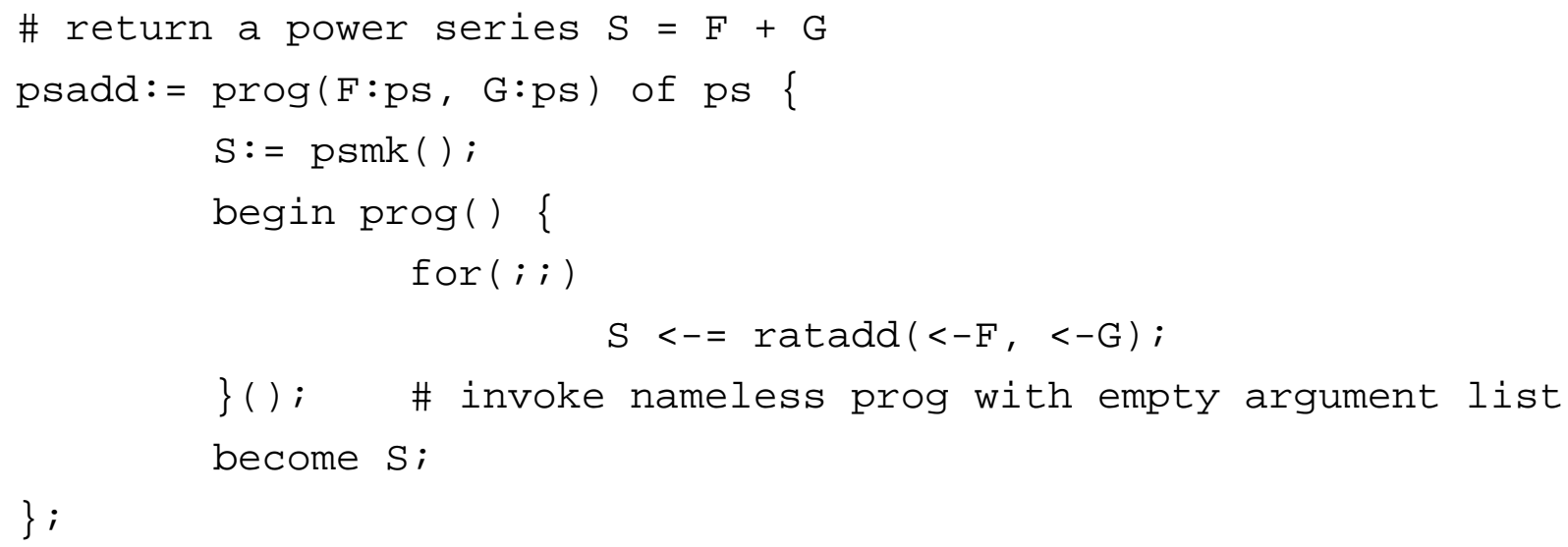

Function psadd creates a power series $S$ to carry the result, starts a process to do the addition, and returns the new power series. In this program the keyword become may be read as a simple "return," although it has a more general meaning, as we shall soon see. The parentheses () after the inner prog are significant: they stand for invocation (with no arguments). The outer prog, by contrast, is not invoked; arguments will be provided later when it is, thus:

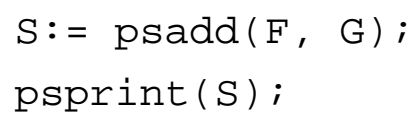

or in shorter fashion:

psprint (psadd $(F, G))$;

We shall need some other basic power series routines.

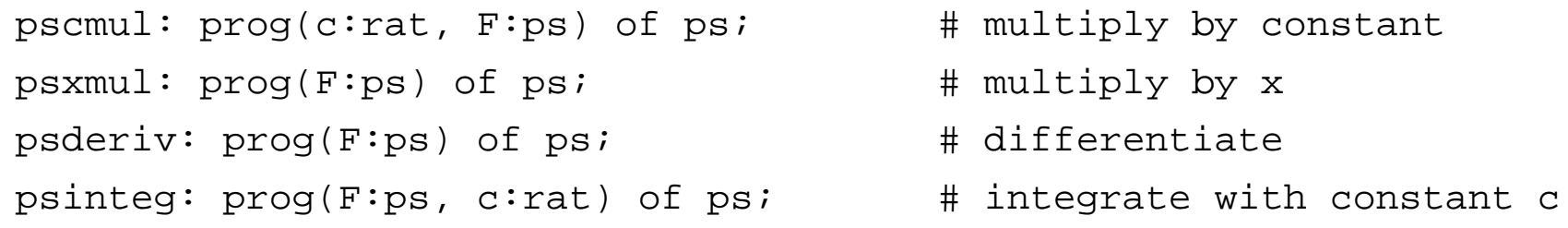

These are all straightforward one-input-one-output flow processes. For example, here is psderiv, programmed in the same style as psadd. 


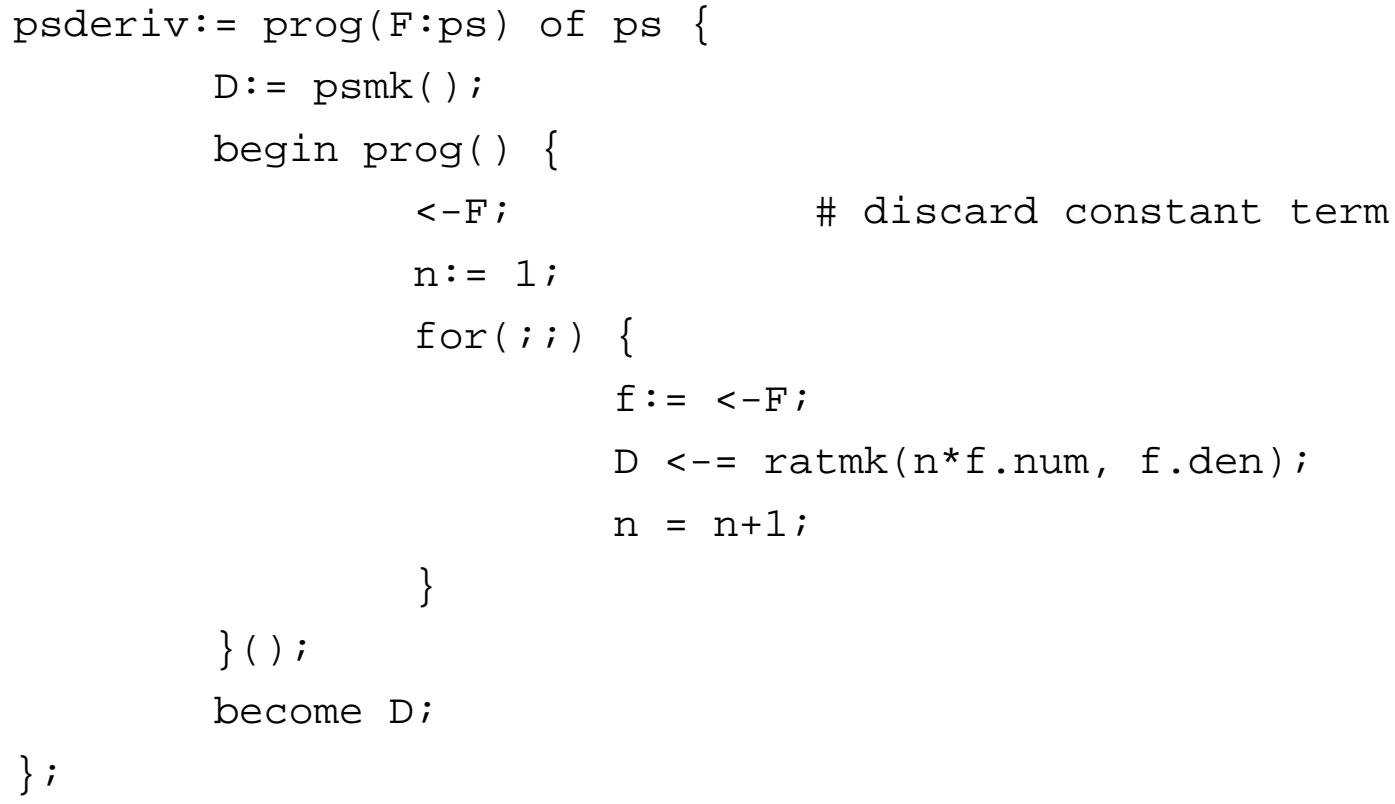

For testing purposes, suppose the power series Ones is an infinite sequence of 1 's-the series for $1 /(1-x)$. A workable definition is

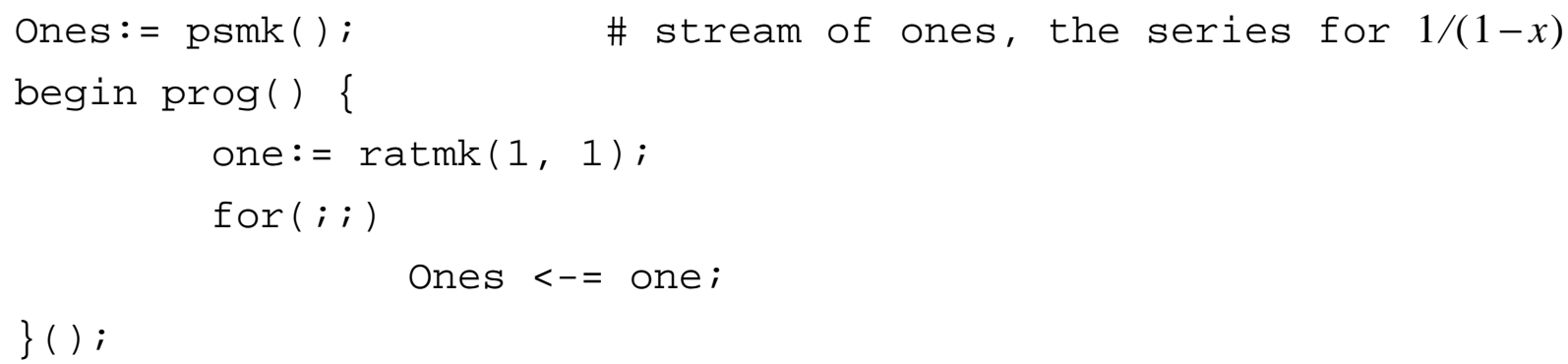

From Ones we can produce other interesting streams:

psprint (psderiv(Ones));

prints $1 \quad 2 \quad 3 \quad 4 \quad 5 \quad \ldots$; and

psprint (psadd (Ones, psxmul (pscmul (ratmk (-1,1), Ones))));

prints Ones $+x \cdot(-1) \cdot$ Ones, or $1 \quad 0 \quad 00000$. This last example is dishonest: some terms of the series Ones are seen only by psadd, the rest by pscmul. It works only because all the terms are the same. To go further we need to be able to split a stream so that each term may be seen by all its consumers.

\section{Splitting a stream}

The routines shown above consume their inputs, leaving nothing for other uses. As we have observed, the more elaborate programs that we wish to build, such as 
multiplication of power series, need fanout. In preparation, we next design a streamsplitting process. Since the two branches of a split stream may be read at different rates, we shall need a queue to hold values to be read later. The queue will materialize as a chain of processes, each holding a single value.

The program begins by reading one item from the input F. Not knowing which of the two outputs will be read first, it waits on a select statement for whichever of the two rendezvous cases happens first.

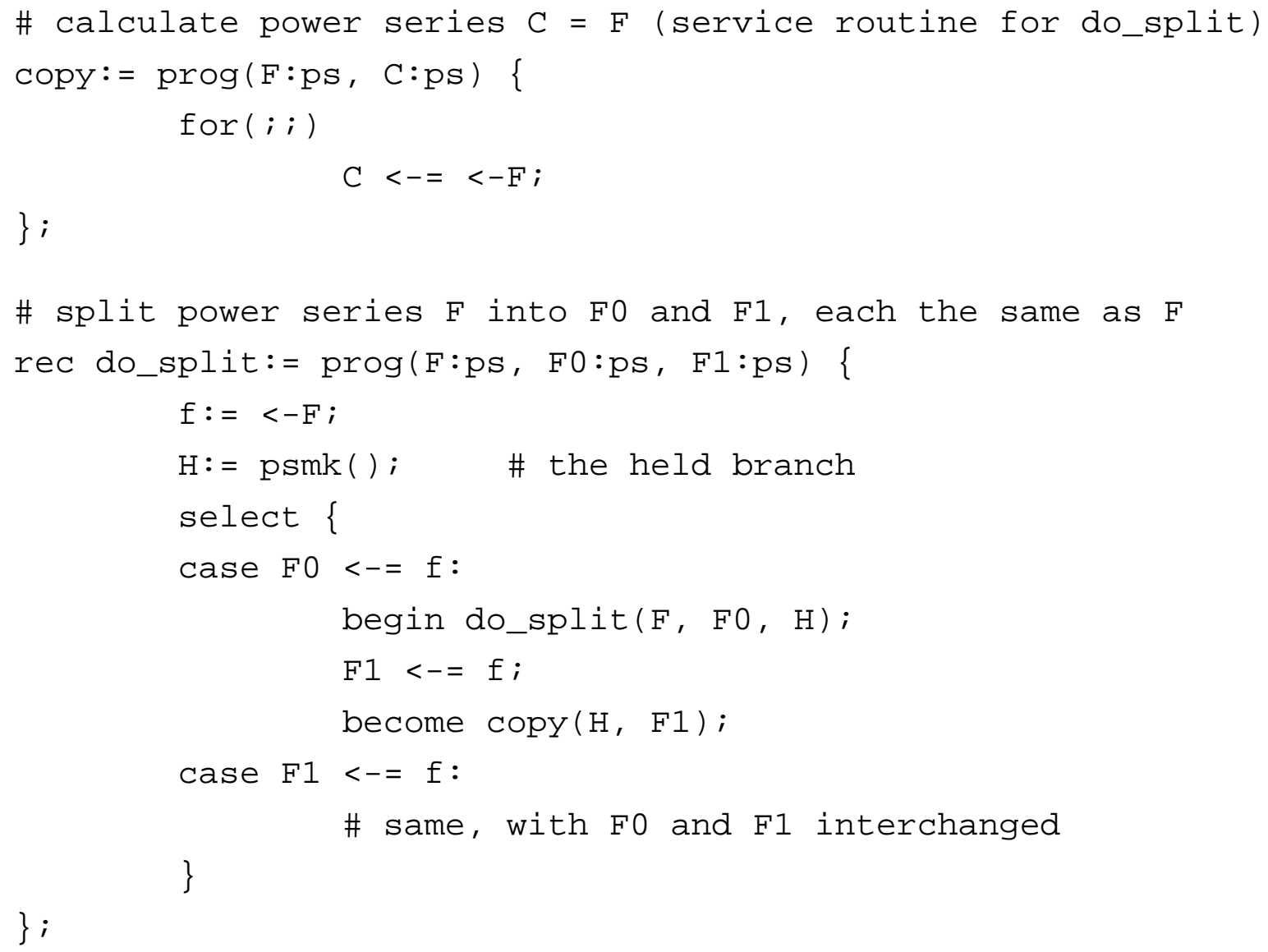

The keyword rec announces that the function is recursive. When the rendezvous on F 0 wins, the previously read item $f$ is sent to $\mathrm{F} 0$ as part of the rendezvous action. The tail of $\mathrm{F}$ is then split recursively in a separate process and the original instance of do_split waits for a rendezvous on the other output stream F1. After that rendezvous, the process is replaced by (becomes) a simple loop that copies from the held queue to $\mathrm{F} 1$.*

Once again it will be convenient to encapsulate the process, this time into a function, split, which takes one power series and returns a pair, declared thus:

* Readers familiar with Hoare's CSP will recognize become as the $\rightarrow$ operator of CSP. ${ }^{1}$ 


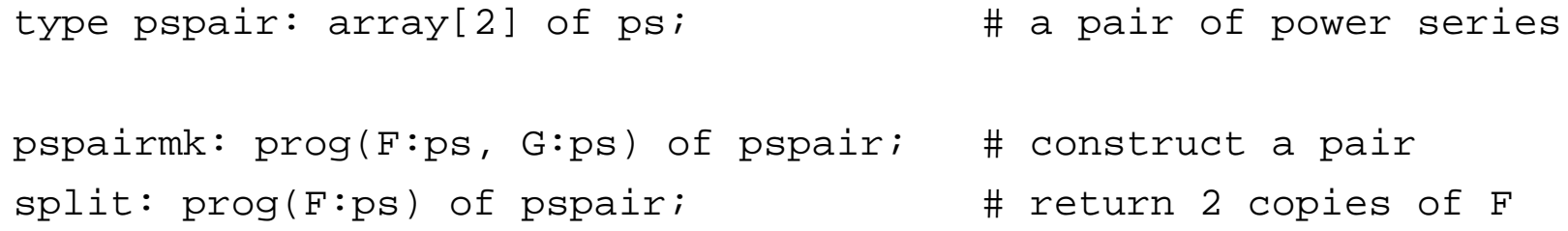

Because its definition is recursive, the name of do_split, unlike that of do_psadd, must persist in the encapsulated version.

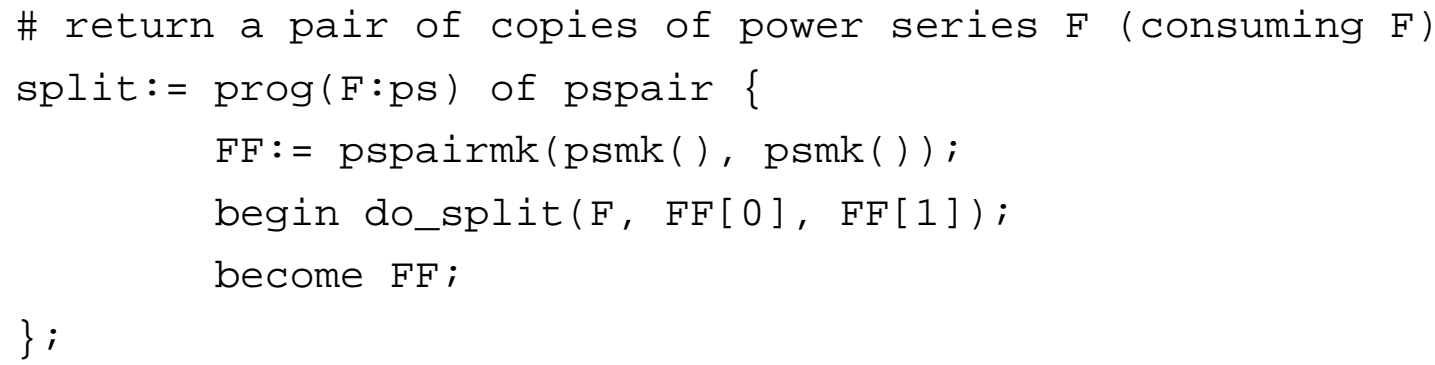

This splitting function has deficiencies, to which we shall return. (You are invited to try to spot them.) Nevertheless, with split, we now have the wherewithal to program a crude version of power series multiplication. Recall the pseudocode,

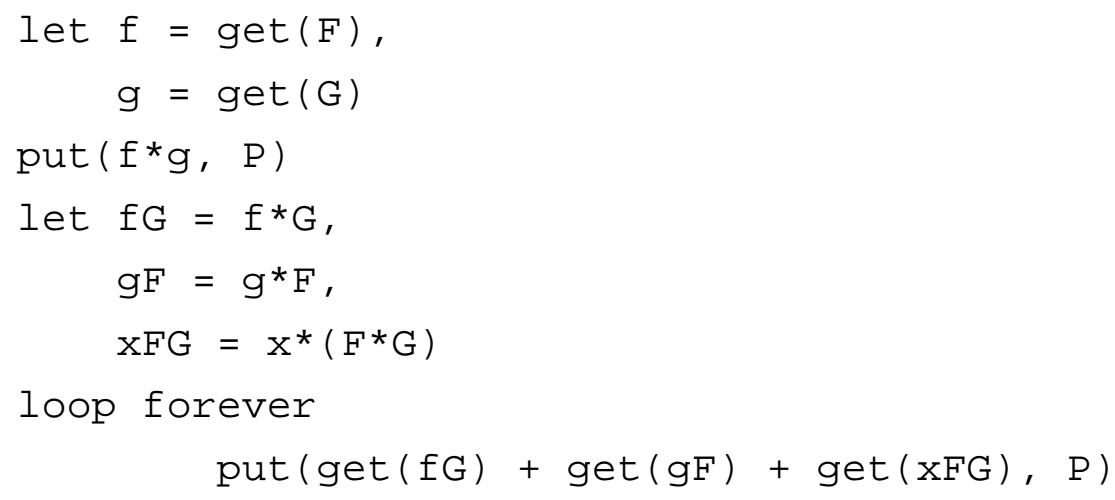

In the newsqueak version that follows, the inner prog implements the pseudocode. The program is encapsulated as usual. 


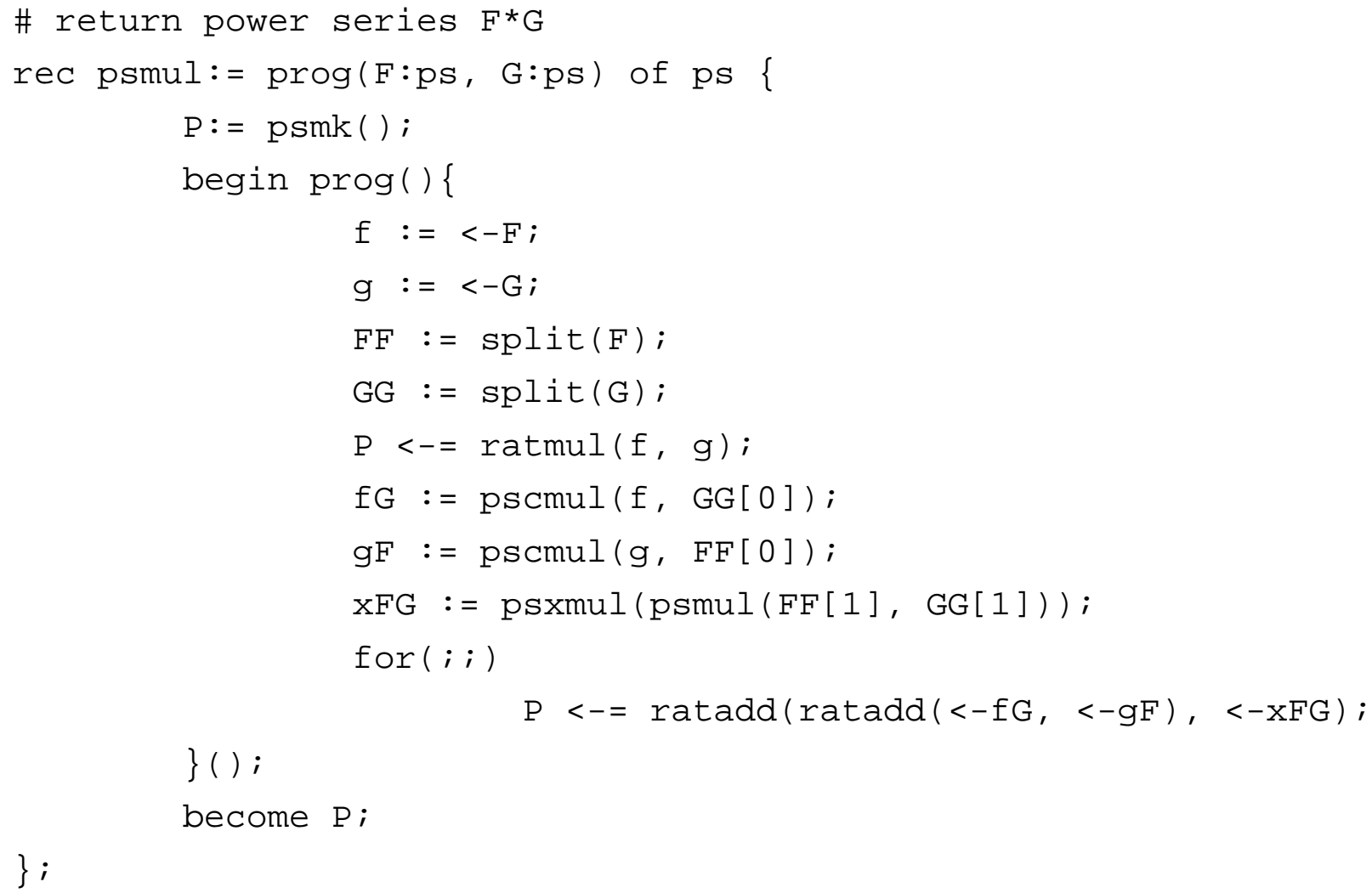

This code works. For example, with Ones defined as before,

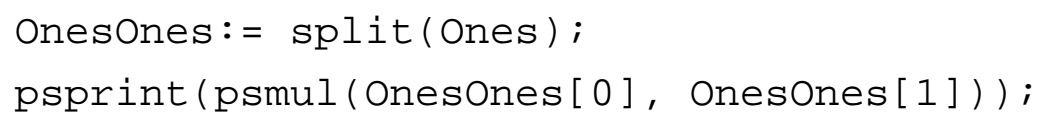

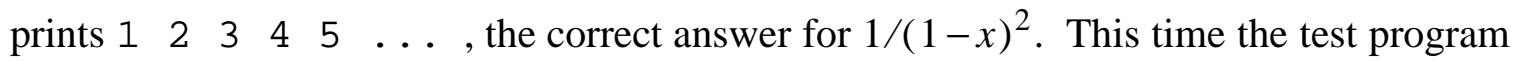
is honest: the stream Ones is split before use. Disappointingly, though, it takes a thousand processes to produce only forty terms - and sluggishly at that. What are all those processes doing?

\section{Reducing overhead}

Figure 2 depicts the recursive decomposition of stream multiplication and its relationship to the convolution formula (1). The $i$ th row contains summands for the $i$ th term of the product $P=F G$. The full figure contains the whole product; the inner triangle contains the product of the tails, $\bar{F} \bar{G}$; and the flanking strips contain the terms of the series $F_{0} \bar{G}$ and $G_{0} \bar{F}$. Stepping down one row corresponds to multiplication by $x$. The four terms in the formula,

$$
F G=F_{0} G_{0}+x F_{0} \bar{G}+x G_{0} \bar{F}+x^{2} \bar{F} \bar{G}
$$




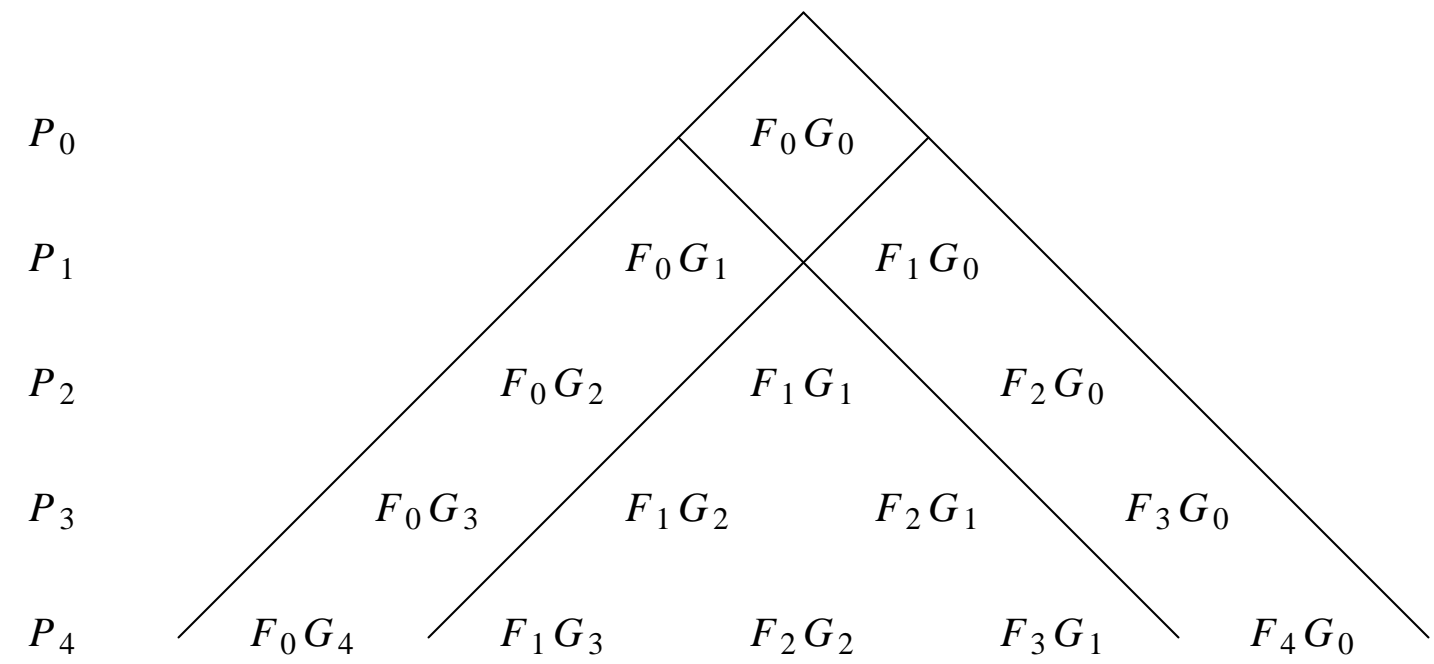

Figure 2. Graphic stream formula for the product $P=F G$. Inner triangle represents $\bar{F} \bar{G}$, left strip $F_{0} \bar{G}$, right strip $G_{0} \bar{F}$.

explain the four-part partition: the first term $F_{0} G_{0}$ at the top, $F_{0} \bar{G}$ and $G_{0} \bar{F}$ at the sides, and the recursive call in the middle.

The side, or tributary, streams, work on freshly split copies of tails of the inputs. To evaluate the $i$ th row, $\lfloor(i+1) / 2\rfloor$ tributaries are needed on each side, each tributary being one term ahead of its inner neighbor. Each term of a split stream is held in a separate incarnation of do_split until it is used. Thereupon the incarnation becomes a copy process, to survive forever after. Thus the number of processes running do_split or copy is the number of off-center entries down through row $i$, or $(i+1)(i+2) / 2-\lfloor i / 2\rfloor$, a quadratic function of the number of tributary streams. By eliminating the copiers we can reduce the process count from quadratic to linear.*

It is easy to direct each held term to the ultimate destination instead of to a chain of copiers. We must, however, preserve sequentiality when one destination is the target of several processes. At any time only the oldest in a chain of held terms may be delivered to that destination; all other processes holding terms must wait. When the oldest process finally delivers the term, it is finished; its last act is to send a signal to notify the next waiting process.

* It would be natural to eliminate copiers by splicing streams. For example, the coda of do_split, namely become copy $(\mathrm{H}, \mathrm{F} 1)$, might be replaced by $\mathrm{F} 1=\mathrm{H}$. Unfortunately, this won't work when parameters are passed by value, as they are in newsqueak. The assignment would not be felt outside do_split in the environment from which F1 originated. 
Processes in the queue may be in four different states, each of which is represented by a named program, with state transitions accomplished by become statements. The four states, or programs, are

do_split. The queue is empty; this is the only process. Both output streams, F0 and $\mathrm{F} 1$, are served.

do_split_new. This is the newest process. It holds no value; all values are held in other processes. Only stream $\mathrm{F} 0$ is served.

do_split_old. This is the oldest process. A value is held; only stream F1 is served.

do_split_mid. This is a waiting process in the middle of the queue. A value is held; no streams are served.

A waiting process will be released by a termination signal from the next older process in the queue. As the signal bears no information aside from the fact of its occurrence, it may be modeled with the "unit" type, which has only one value. As it is useless to supply a value, the signal may be sent on a channel of this type by a simple postfix operator, $<-$, instead of the more elaborate infix operator $<-=$.

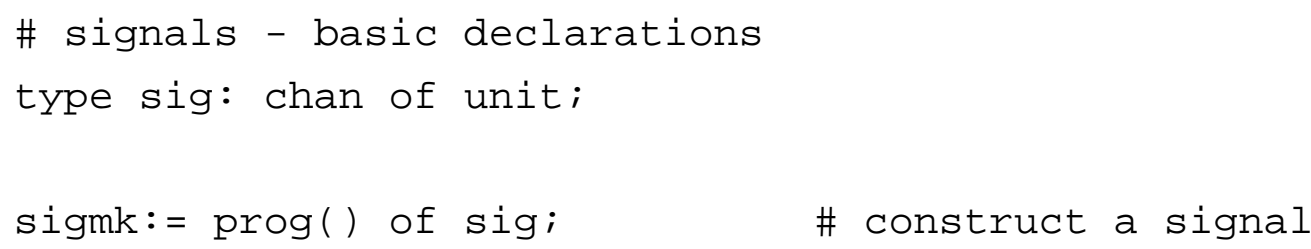

A process in the oldest state needs a held value $f$, the output destination $F 1$, and a release signal to notify the next process when it should proceed.

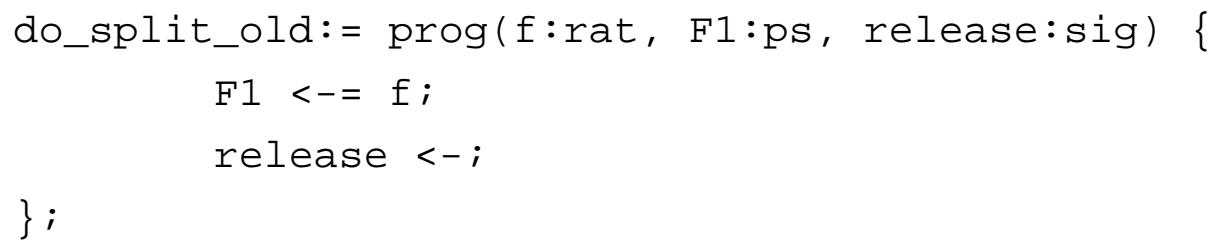

A middle process needs the same information, for it will eventually become oldest. It also needs a signal to wait on. When the signal arrives, the process becomes oldest.

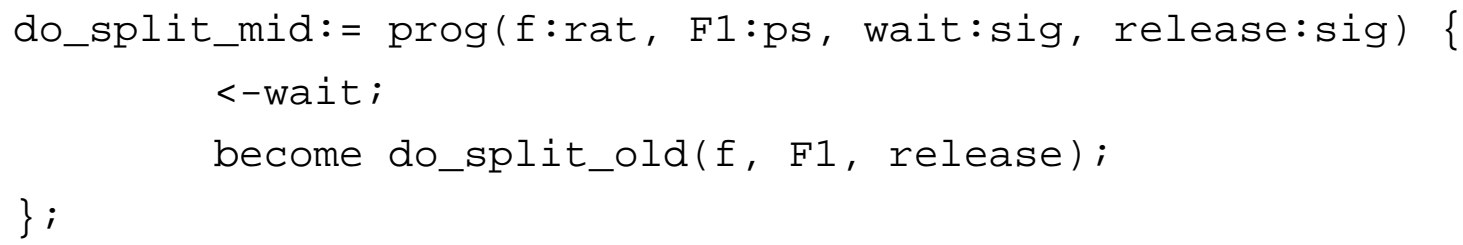


When the queue is empty, there is only one process. It accepts a rendezvous with either output channel. When rendezvous occurs, a new process is begun to serve that channel. The original process, now the oldest, turns to serve the other channel. A signal is provided for the oldest process to announce termination.

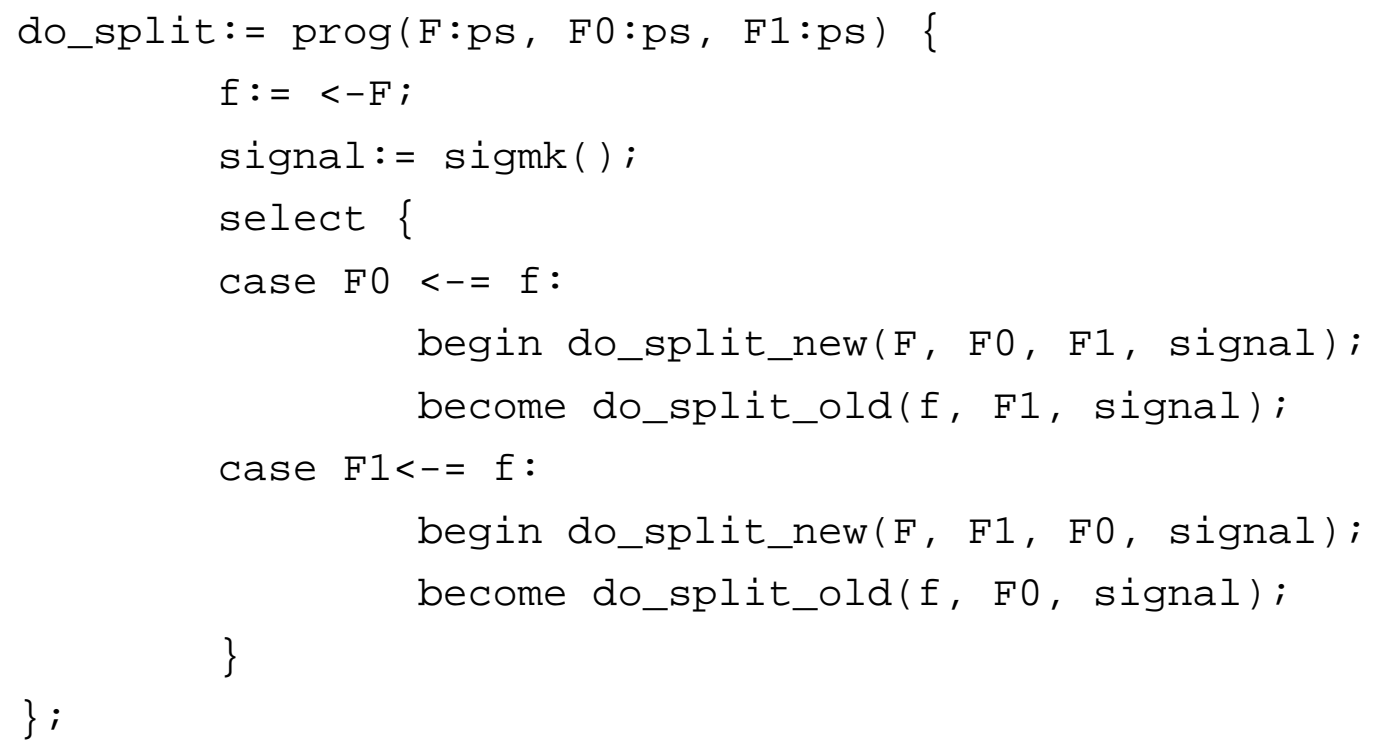

When the queue is not empty, the newest process serves only one output stream, $\mathrm{F} 0$. It must be prepared for two possible occurrences: a demand on that stream, or the emptying of the queue. If the queue becomes empty, the process reverts to the original do_split state. In the other case, the process begins a fresh newest process and itself enters the queue as a middle process.

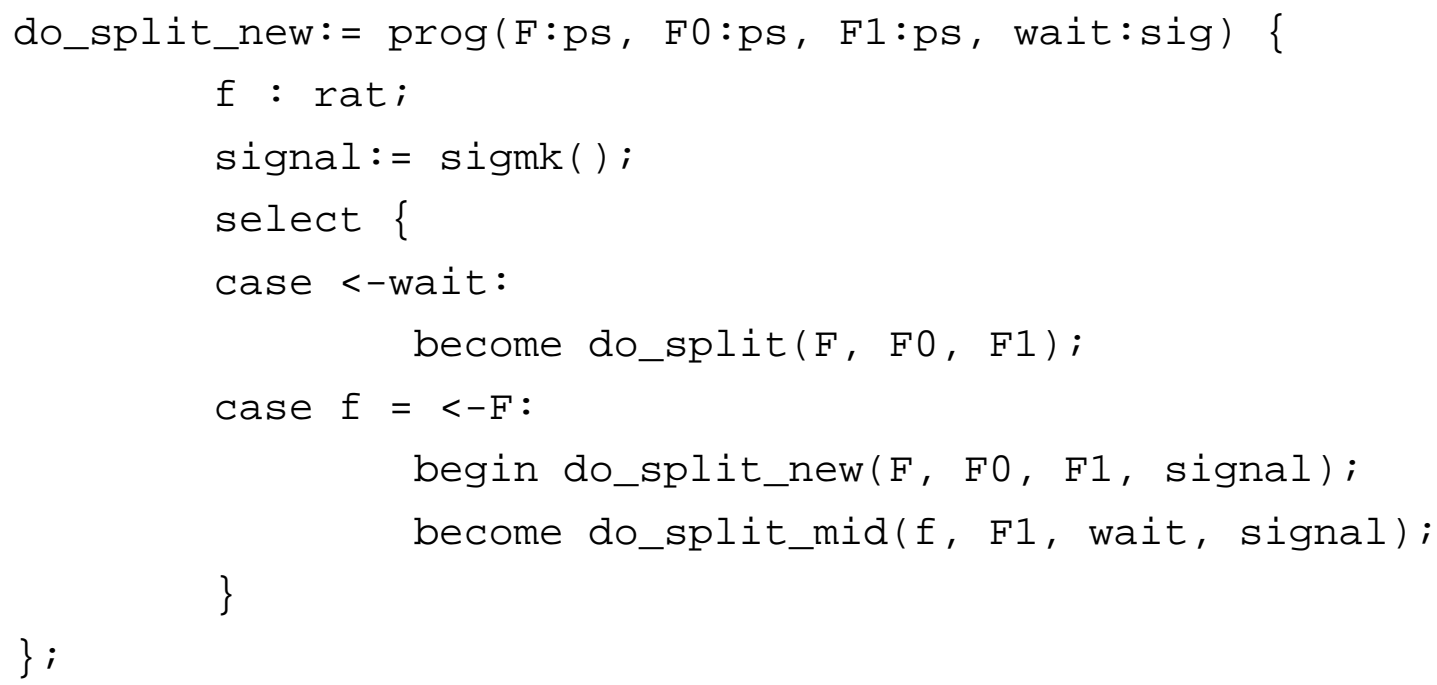

In the preceding code, the apparent recursion via become statements is merely continuation or state transition. There is no stack growth, as there might be if the 
become statements were replaced by procedure calls. Only the programs invoked by begin require new storage; and that storage serves a useful purpose: holding queued values. The four program states are mutually recursive. Gathered into a single newsqueak program, and encapsulated as before, they look like this:

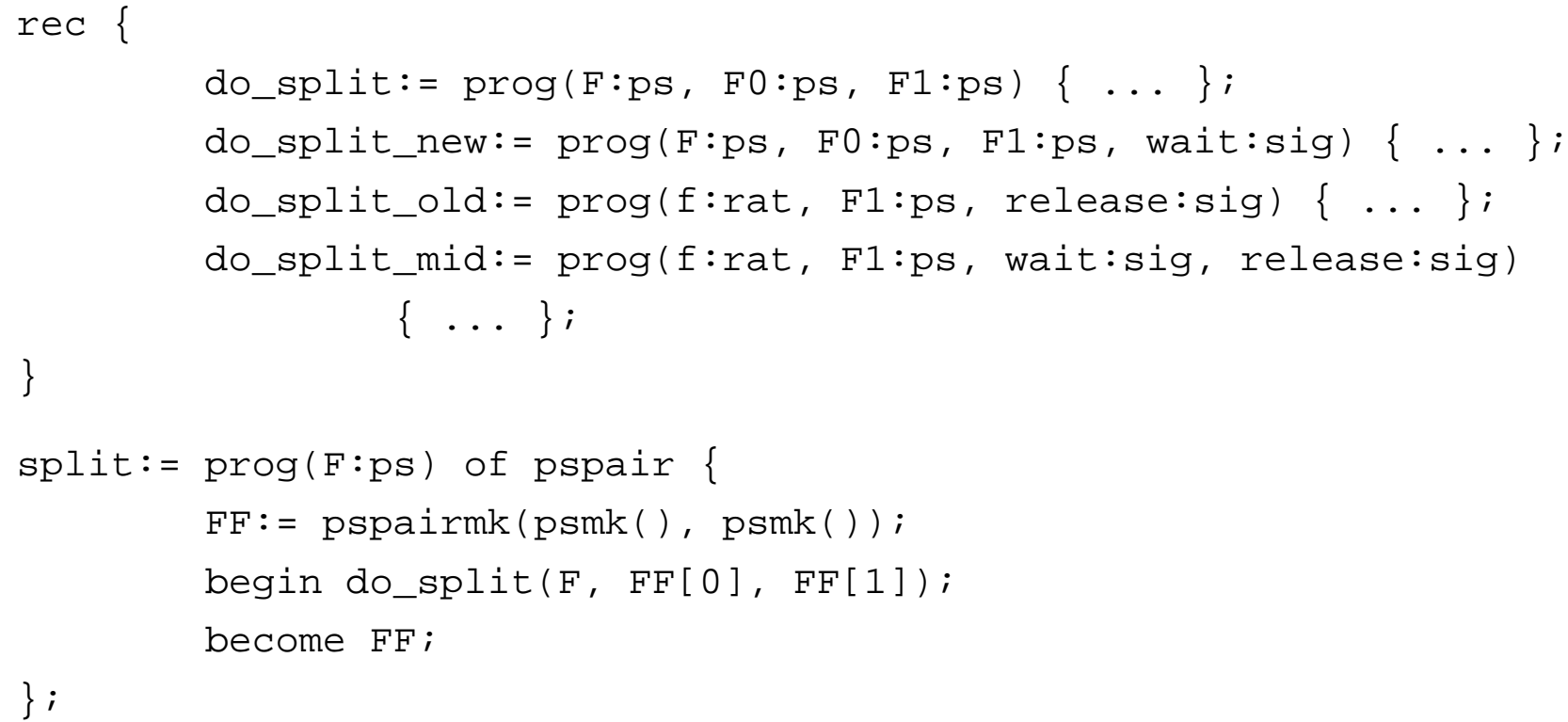

With quadratic splitting replaced by linear, the test case that printed only 40 terms before now prints 168 terms before exhausting a supply of 1000 processes. This is still not very good. Our analysis has shown that to print $i$ terms of a product, we need about $i$ tributary streams made by pscmul, and about $i / 2$ recursive calls to psmul. Each tributary stream is the result of a split. At any time each split comprises one head process plus one process per held value. Each tributary stream is split from its predecessor and is one step behind. Thus it must engage two processes, one for splitting and one for holding. Finally, for each recursive call there is one psxmul process. Altogether about $4 i$ processes are needed to compute $i$ terms; 1000 processes might be expected to compute 250 terms.

The difference between 250 and the observed value of 168 is accounted for by runaway incarnations of do_split producing values ahead of needs. The amount of runaway depends on the exact order in which the multiprocess computation has proceeded.

Although 250 terms may seem like an enormous number, power-series expressions with $n$ multiplications may be expected to engage $n$ times as many processes, and so run out of space much sooner. Moreover, as we shall see, other power series operations have a much larger appetite for processes than does multiplication. To be prepared for 
expressions of more complexity, we cannot use processes recklessly. Runaways are the major remaining profligacy; to prevent them we must attend to scheduling.

\section{Demand channels}

We wish to build a scheduling protocol that forces lazy evaluation. Processes will compute output values only on demand, and will not demand inputs or start other processes until they are needed. This we accomplish by redefining power series as twoway "demand channels," which comprise request and data channels.

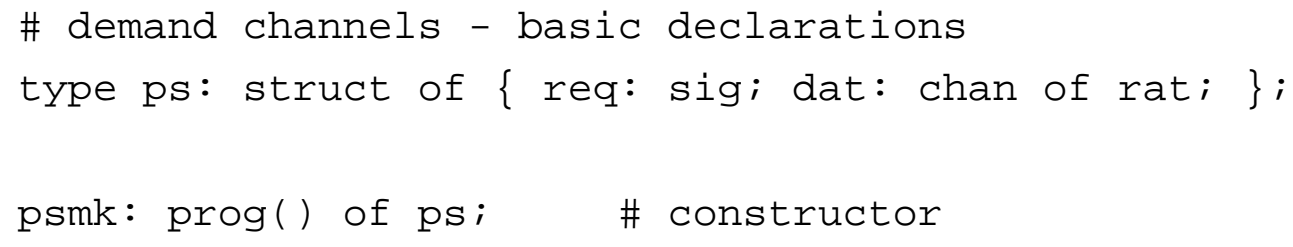

The protocol of a demand channel is exemplified by the following primitive subroutine to get a term from a power series: send a signal on the request channel to stimulate computation of the term and then receive the term from the data channel.

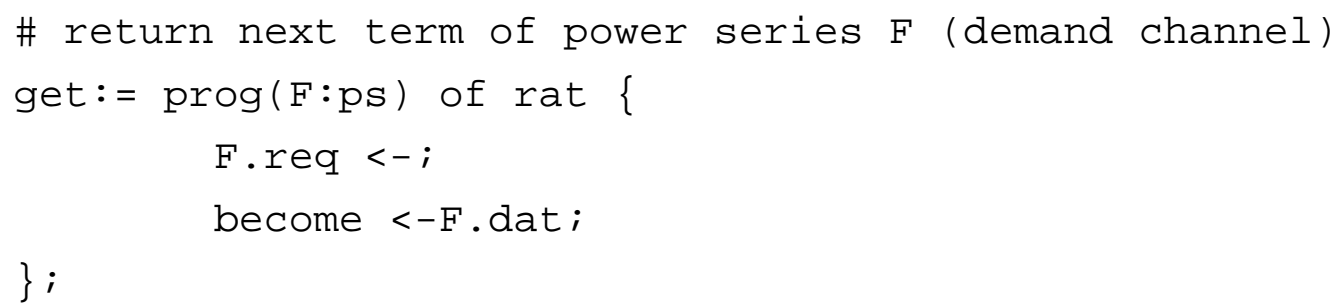

Recoding for demand channels is a straightforward matter. Each computation that needs input must be guarded by a receipt from the output request channel. Inputs are obtained with get. For example, in the power series adder, just one statement,

$$
\mathrm{S}<-=\operatorname{ratadd}(<-\mathrm{F},<-\mathrm{G}) \text {; }
$$

must be changed, to

$$
\begin{aligned}
& <- \text { S.req; } \\
& \text { S.dat }<-=\operatorname{ratadd}(\operatorname{get}(F), \operatorname{get}(G)) \text {; }
\end{aligned}
$$

With all functions similarly recoded for demand channels, the test case,

$$
\text { psprint (psmul (Onesones [0], Onesones [1])); }
$$

prints 248 terms before exhausting a supply of 1000 processes. According to the previous analysis, which showed that each term requires at least 4 new processes, the 
scheduling is optimal. To improve matters further, we must reduce the per-term requirements.

The newest in a chain of do_split processes does not pull full weight, for it contains no held value. For this reason, with optimal scheduling, every tributary stream in psmul was observed to engage two processes. The two processes may be reduced to one by letting the newest process hold the first value and distinguishing two states of the process, one "full" and one "empty." Furthermore, by coding psxmul in-line in psmul, a further process may be avoided for each recursion level, or one for each two output terms. At the outer level of Figure 1, there would remain one process for the enclosing box, one for each of the left and right inner boxes, and a splitting process for each of the two inputs. These improvements will bring the process count down to 2.5 per output term.

\section{Other operations}

Substitution of power series. We have already seen (3) that the substitution $S=F(G)$, where $G_{0}=0$, may be computed from the formula

$$
S=S_{0}+x \bar{S}=F_{0}+x \bar{G} \bar{F}(G) .
$$

The corresponding newsqueak code is

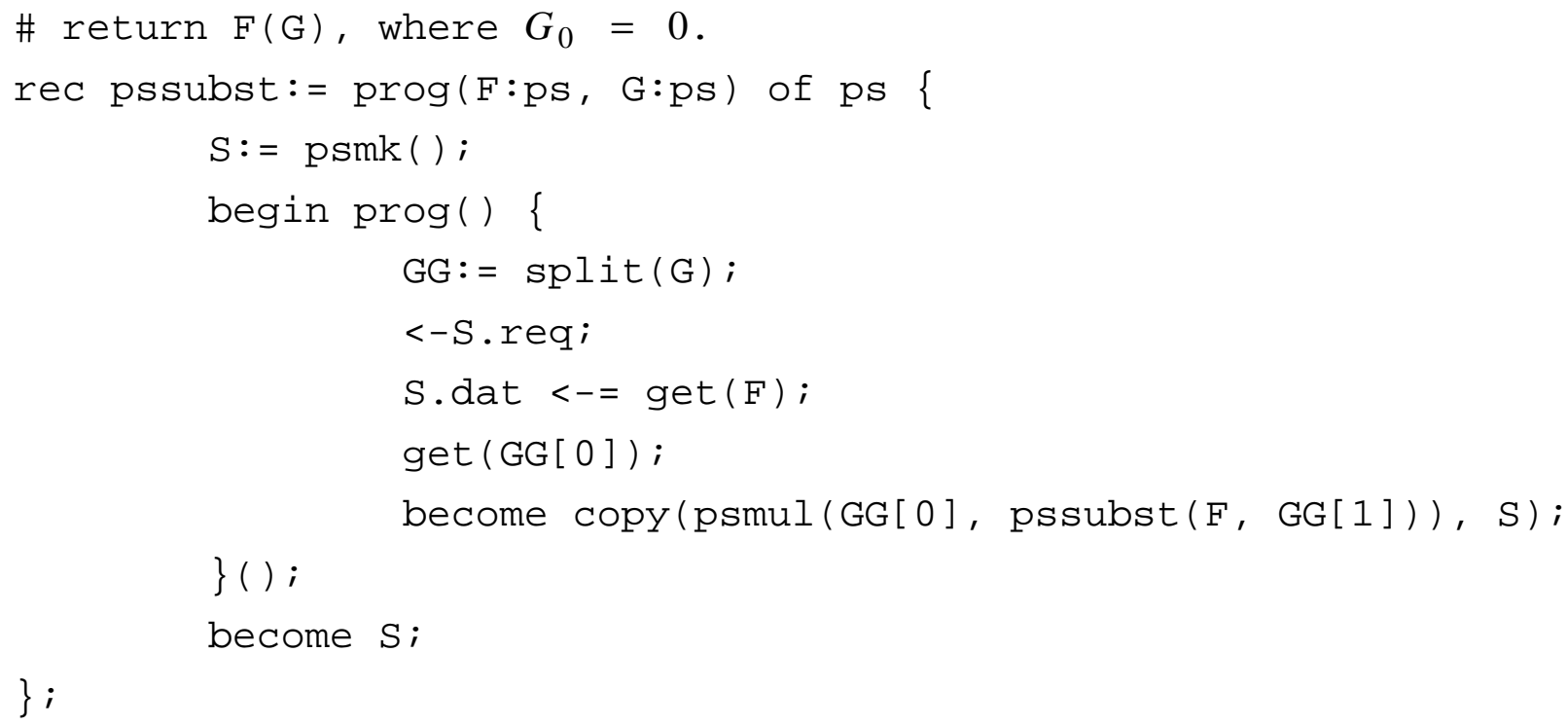

This code, like the original do_split, leaves a copier at every stage of recursion. The copiers here, however, are not cascaded, so there is only a constant, rather than an $O(n)$, factor to be gained from eliminating them. 
Exponential of a power series. From the previously derived pseudocode,

$$
\begin{aligned}
\text { let } D & =\operatorname{deriv}(F) \\
P & =X \star D \\
I & =\operatorname{integ}(P, 1) \\
\text { loop forever } & \operatorname{put}(\operatorname{get}(I), X)
\end{aligned}
$$

comes the following newsqueak program. The call for copy is no cause for alarm, because there is no recursion.

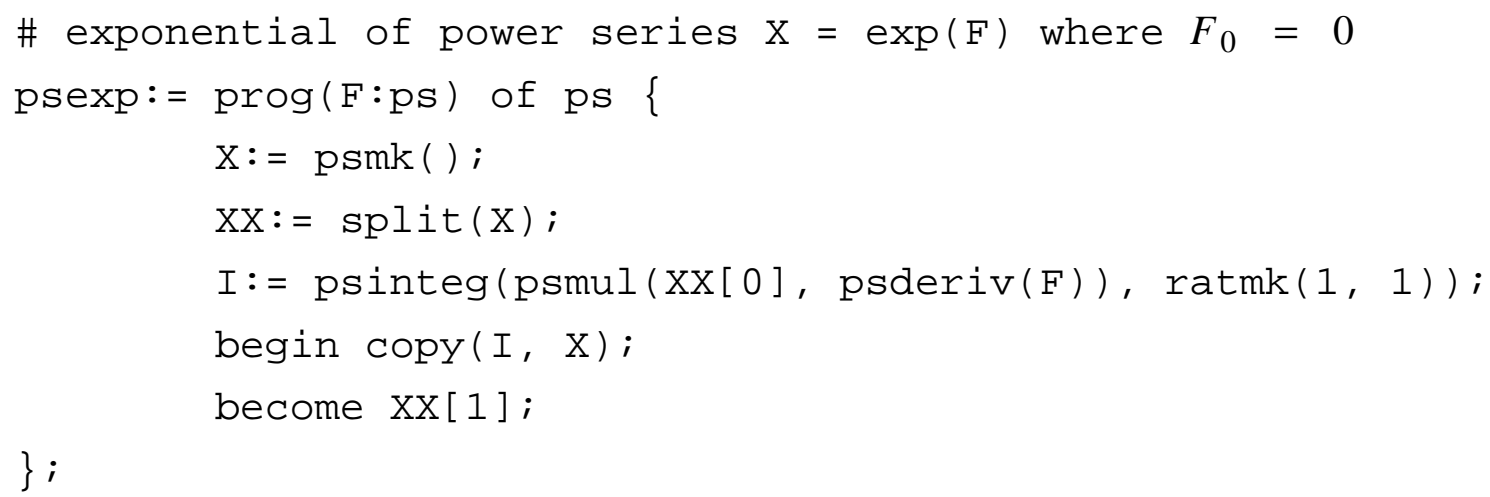

Reciprocal of a power series; find $R$ such that $F R=1$. Expand $F$ to obtain

$$
\left(F_{0}+x \bar{F}\right) R=1,
$$

or

$$
R=R_{0}+x \bar{R}=\frac{1}{F_{0}}(1-x \bar{F} R) .
$$

From this formula we see that the reciprocal has the same computational complexity as multiplication. We may also read off a translation into newsqueak. 


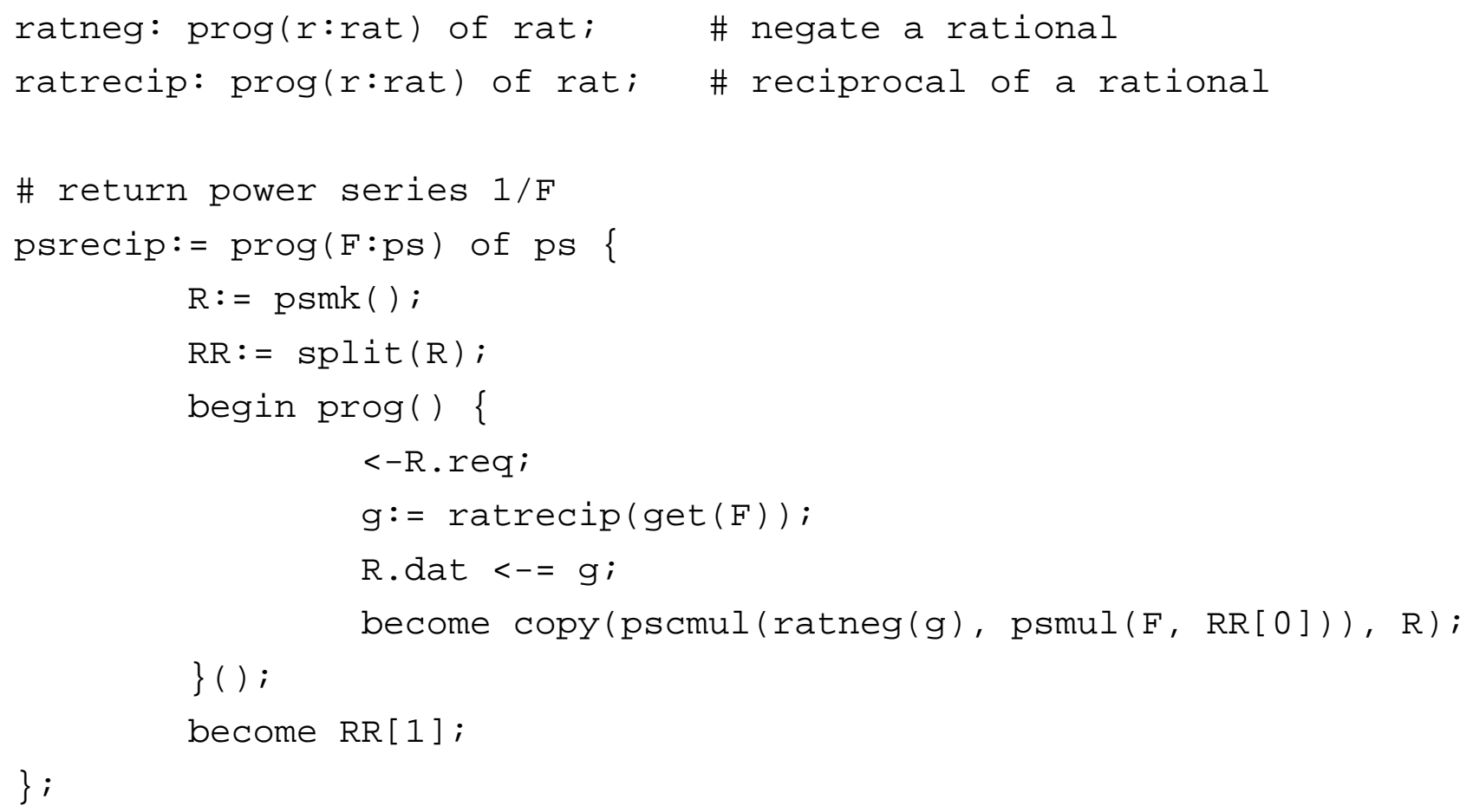

Reversion of power series. Find the functional inverse of power series $F$. That is, find $R$ such that

$$
F(R(x))=x
$$

From equating coefficients we find that $R_{0}$ must satisfy

$$
\sum_{i=0}^{\infty} F_{i} R_{0}^{i}=0
$$

that is, $R_{0}$ must be a root of $F$. To keep the coefficients of $R$ rational, we stipulate that $R_{0}=0$. Hence also $F_{0}=0$. Thus we assume that $F$ has the form

$$
F(x)=x \bar{F}=x F_{1}+x^{2} \overline{\bar{F}}
$$

and similarly for $R$. The basic identity (7) becomes

$$
x \bar{R} \bar{F}(R)=x
$$

Expand $F$ and solve for $\bar{R}$ (which may also be written $R_{1}+x \overline{\bar{R}}$ ).

$$
\begin{aligned}
& x \bar{R}\left(F_{1}+x \bar{R} \overline{\bar{F}}(R)\right)=x, \\
& \bar{R}=R_{1}+x \overline{\bar{R}}=\frac{1}{F_{1}}\left(1-x \bar{R}^{2} \overline{\bar{F}}(R)\right) .
\end{aligned}
$$


Since $R_{0}$ is zero, the substitution $\overline{\bar{F}}(R)$ satisfies the precondition of pssubst, and that procedure may be used. Counting $\bar{R}^{2}$ as two appearances, $R$ appears three times in the right side of (8); the newsqueak program must involve three splits. Otherwise it is like all the rest, and so will be omitted.

As an example of the use of reversion, consider calculating $\tan (x)$ from its simpler inverse function $\arctan (x)$. The $\operatorname{arctangent}$ has an easy derivative,

$$
\frac{d}{d x} \arctan (x)=\frac{1}{1+x^{2}}
$$

Posit a monomial substitution operator, psmsubst $(\mathrm{F}, \mathrm{C}, \mathrm{n})$, that calculates $F\left(c x^{n}\right)$.* Substitute $-x^{2}$ into Ones (i.e. into $\left.1 /(1-x)\right)$ to get $1 /\left(1+x^{2}\right)$, integrate to get the arctangent, and revert. The resulting code,

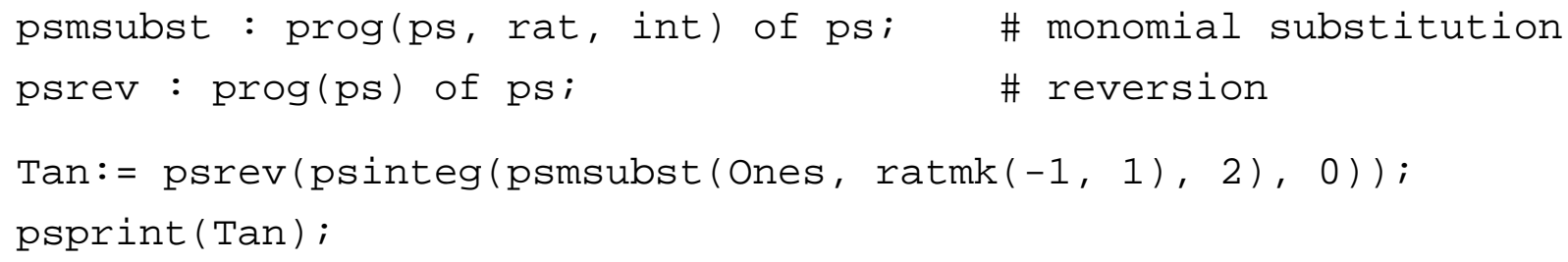

prints the coefficients of the tangent series:

\section{$\begin{array}{llllllllllllll}0 & 1 & 0 & 1 / 3 & 0 & 2 / 15 & 0 & 17 / 315 & 0 & 62 / 2835 & 0 & 1382 / 155925 & \ldots\end{array}$}

Various algorithms and formulas for reversion may be found in the literature. ${ }^{10,11}$ None that I have seen are as straightforward as (8), which completely hides the combinatoric complexity, yet constitutes a detailed specification for a program. One could not ask for a better testimonial for stream methods.

\section{Complexity}

The complexity of the several stream operations may be derived from their defining equations. For definiteness, we shall count the number of coefficient-domain products necessary to compute coefficients of $x^{0}, x^{1}, \ldots, x^{n}$ in an output series.

Multiplication. Let $\operatorname{Prod}(n)$ denote the desired count. From (6)

$$
P=F G=F_{0} G_{0}+x F_{0} \bar{G}+x G_{0} \bar{F}+x^{2} \bar{F} \bar{G} .
$$

To compute terms $P_{0}$ through $P_{n}$, we need

* This simple operator multiplies each input coefficient $F_{i}$ by $c^{i}$ and copies it to the output followed by $n-1$ zeros. 
the single product $F_{0} G_{0}$,

if $n \geq 1$, terms of $F_{0} \bar{G}$ and $G_{0} \bar{F}$ through $x^{n-1}$, and

if $n \geq 2$, terms of $\bar{F} \bar{G}$ through $x^{n-2}$.

Thus $\operatorname{Prod}(n)$ satisfies

$$
\begin{aligned}
& \operatorname{Prod}(0)=1 \\
& \operatorname{Prod}(1)=3 \\
& \operatorname{Prod}(n)=1+2 n+\operatorname{Prod}(n-2), \quad n \geq 2,
\end{aligned}
$$

or, in closed form,

$$
\operatorname{Prod}(n)=\left(\begin{array}{c}
n+2 \\
2
\end{array}\right)=O\left(n^{2}\right)
$$

Thus, if rational operations are counted as unit time, stream multiplication takes quadratic time. Space, as measured by the process count, is also linear.

Substitution. From (3), we have

$$
S=F(G)=F_{0}+x \bar{G} \bar{F}(G) .
$$

To compute $S_{0}$ through $S_{n}$, where $n \geq 1$, we need

terms of $\bar{F}(G)$ through $x^{n-1}$, and terms of the product $\bar{G} \bar{F}(G)$ through $x^{n-1}$,

whence the desired count, $\operatorname{Subst}(n)$, satisfies

$$
\begin{aligned}
& \operatorname{Subst}(0)=0 \\
& \operatorname{Subst}(n)=\operatorname{Subst}(n-1)+\operatorname{Prod}(n-1), \quad n \geq 1,
\end{aligned}
$$

with the closed form solution

$$
\operatorname{Subst}(n)=\left(\begin{array}{c}
n+2 \\
3
\end{array}\right)=O\left(n^{3}\right)
$$

Reversion. From (8), the solution $R$ of $F(R(x))=x$ satisfies

$$
R=\frac{x}{F_{1}}\left(1-x \bar{R}^{2} \overline{\bar{F}}(R)\right)
$$

To compute $R_{0}$ through $R_{n}$, where $n \geq 2$, we need 
terms of $\overline{\bar{F}}(R)$ through $x^{n-2}$,

terms of two power series products through $x^{n-2}$, and

$n-1$ termwise multiplications by $1 / F_{1}$.

The desired count, $\operatorname{Rev}(n)$, satisfies

$$
\begin{aligned}
& \operatorname{Rev}(0)=\operatorname{Rev}(1)=0, \\
& \operatorname{Rev}(n)=n-1+2 \operatorname{Prod}(n-2)+\operatorname{Subst}(n-2), \quad n \geq 2 .
\end{aligned}
$$

Substituting and simplifying, we find that reversion and substitution are equally difficult in this measure:

$$
\operatorname{Rev}(n)=\left(\begin{array}{c}
n+2 \\
3
\end{array}\right)-1=O\left(n^{3}\right), n \geq 1 .
$$

Exponentiation, reciprocation. Both operations require $O\left(n^{2}\right)$ coefficient-domain products.

\section{Discussion}

The method here demonstrated calculates the coefficients of power series defined by sets of recursive equations, provided the equations express later terms in terms of earlier ones. We have seen how such recursive equations can be translated straightforwardly into stream-processing programs. In doing the translation, two technical difficulties were encountered. The first, the necessity for fanout and queuing, was solved by a splitting function. The second, minimizing computation in excess of the needs of the ultimate consumer, was solved by demand channels.

The stream algorithms realize the same complexity as other power series algorithms with the "sequential" property of producing $n$ terms of output without accessing more than $n+k$ terms of input for some fixed $k .{ }^{11}$ Although more efficient nonsequential algorithms are known, ${ }^{12}$ the stream formulation remains attractive by reason of its extreme simplicity. The availability of such programming techniques would simplify the organization of symbolic calculations, which are central in systems like Macsyma or Maple, and are beginning to make their way into numerical algorithms.

The style of programming used here was first articulated by Landin, who described a data stream in applicative terms as a function that returns a pair comprising the first element of the stream and a continuation. The continuation is another function of the same type. ${ }^{13}$ To get the second element, invoke the continuation. It will deliver another continuation for further reference, and so on. Channels may be understood as Landin 
stream variables. A process receiving from a channel effectively invokes the continuation and stores the continuation part of the result back into the channel. The corresponding sender, however, is at liberty to calculate ahead in preparation for the next rendezvous. Herein lies the potential for runaway, which was cured by using demand channels. Demand channels enforce the most literal interpretation of the Landin model, where future needs are never anticipated.

Besides giving us a way to describe stream processes, the Landin model gives us a way to think about them. The first-plus-tail style of mathematical analysis, as a perfect match to the first-plus-continuation model of streams, leads directly to useful and otherwise nonobvious stream algorithms. First-plus-tail analysis, of course, may be used in stream applications other than power series. For example, Kahn and MacQueen mention unlimited-precision computation with reals, where streams carry the digits. ${ }^{4}$ In a domain of infinite sequences, recursive equations are a mode of expression as natural as arithmetic formulas are in a scalar domain.

As we have seen, infinite lists, which underlie lazy evaluation systems, constitute an alternative to data stream representations. Recursive equations lead somewhat more directly to programs on infinite lists than on channels, as lazy-evaluation systems already contain the infrastructure to handle fanout and scheduling. But once the infrastructure is built and hidden, programs in the two kinds of systems look quite similar. The newsqueak program for the tangent,

Tan:= psrev (psinteg (psmsubst (Ones, ratmk $(-1,1), 2), 0))$; looks just as it would in any other functional language. For comparison, I programmed the application in ML, ${ }^{14}$ using a lazy-stream module written by David MacQueen. The ML source code was about half the size of the newsqueak.* However, the compiled ML ran only one-fifth as fast as the interpreted newsqueak. Channels make a difference.

As stream processing makes its way into mainstream languages, the style of programming illustrated here will become increasingly important.

* The following example of the ML code may be compared with the reciprocal operator given under “'Other operations"' above. The notation $\mathrm{fn}()=>$ introduces a lambda expression for a pair-valued Landin function.

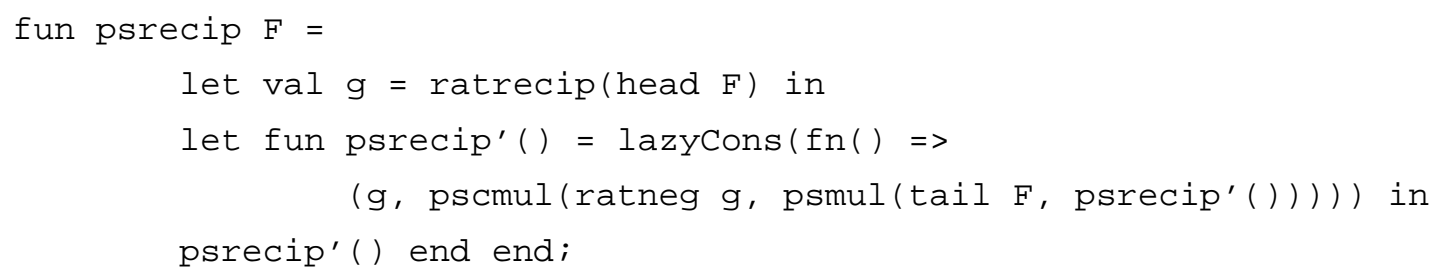


I am grateful to Gilles Kahn for introducing me to this elegant application, to Jon Bentley for bringing me up to date on complexity matters, to Dave MacQueen for semantic insight and assistance with ML, and to Rob Pike for tuning squint to meet the stresses of the application and for thoughtful advice on drafts of this paper. 


\section{References}

[1] Hoare, C. A. R., Communicating Sequential Processes, Prentice-Hall, Englewood Cliffs, NJ (1985).

[2] Hehner, E. C. R., Logic of Programming, Prentice-Hall (1984).

[3] Pike, R., "The Implementation of Newsqueak," Software(emPractice and Experience (this issue).

[4] Kahn, G. and MacQueen, D. B., "Coroutines and Networks of Parallel Processes,' Information Processing 77, Proceedings of IFIP Congress 77 7, pp. 993-998, Gilchrist, B. (Ed.), North-Holland, Amsterdam (1977).

[5] Ford, L. R., Differential Equations, McGraw-Hill (1955).

[6] Abelson, H. and Sussman, G. J., Structure and Interpretation of Computer Programs, MIT Press, Cambridge, MA (1985).

[7] Turner, D., “An Overview of Miranda," ACM SIGPLAN Notices 21(12) (December, 1986).

[8] Wikstrom, A., Functional Programming Using Standard ML, Prentice-Hall, Englewood Cliffs, NJ (1987).

[9] Pike, R., “Newsqueak: A Language for Communicating with Mice,' CSTR 143, AT\&T Bell Laboratories, Murray Hill, NJ (1989).

[10] Van Orstrand, C. E., "Reversion of Power Series,' London, Edinburgh, and Dublin Philosophical Magazine 19(109), pp. 366-376 (1910).

[11] Knuth, D. E., The Art of Computer Programming, Vol. 2, Addison-Wesley, Reading, MA (1969). §4.7.

[12] Brent, R. P. and Kung, H. T., "Fast Algorithms for Manipulating Formal Power Series,' JACM 25, pp. 581-595 (1978).

[13] Landin, P. J., “A Correspondence between ALGOL 60 and Church's Lambda Notation, Part I,' CACM 8, pp. 89-101 (1965).

[14] Harper, R., Milner, R., and Tofte, M., The Definition of Standard ML, MIT Press (1990). 